\title{
Experimental Realization of Einstein-Podolsky-Rosen-Bohm Gedankenexperiment: A New Violation of Bell's Inequalities
}

\author{
Alain Aspect, Philippe Grangier, and Gérard Roger \\ Institut d'Optique Théorique et Appliquée, Laboratoire associé au Centre National de la Recherche Scientifique, \\ Université Paris-Sud, F-91406 Orsay, France
}

(Received 30 December 1981)

The linear-polarization correlation of pairs of photons emitted in a radiative cascade of calcium has been measured. The new experimental scheme, using two-channel polarizers (i.e., optical analogs of Stern-Gerlach filters), is a straightforward transposition of Einstein-Podolsky-Rosen-Bohm gedankenexperiment. The present results, in excellent agreement with the quantum mechanical predictions, lead to the greatest violation of generalized Bell's inequalities ever achieved.

PACS numbers: $03.65 . \mathrm{Bz}, 35.80 .+\mathrm{s}$

In the well-known Einstein-Podolsky-RosenBohm gedankenexperiment ${ }^{1}$ (Fig. 1), a source emits pairs of spin- $\frac{1}{2}$ particles, in a singlet state (or pairs of photons in a similar nonfactorizing state). After the particles have separated, one performs correlated measurements of their spin components along arbitrary directions $\vec{a}$ and $\vec{b}$. Each measurement can yield two results, denoted

$$
E(\overrightarrow{\mathrm{a}}, \overrightarrow{\mathrm{b}})=P_{++}(\overrightarrow{\mathrm{a}}, \overrightarrow{\mathrm{b}})+P_{--}(\overrightarrow{\mathrm{a}}, \overrightarrow{\mathrm{b}})-P_{+-}(\overrightarrow{\mathrm{a}}, \overrightarrow{\mathrm{b}})-P_{-+}(\overrightarrow{\mathrm{a}}, \overrightarrow{\mathrm{b}})
$$

is the correlation coefficient of the measurements on the two particles. Bell ${ }^{2}$ considered theories explaining such correlations as due to common properties of both particles of the same pair; adding a locality assumption, he showed that they are constrained by certain inequalities that are not always obeyed by the predictions of quantum mechanics. Such theories are called" "realistic local theories" and they lead to the generalized Bell's inequalities ${ }^{4}$

$$
-2 \leqslant S \leqslant 2,
$$

where

$$
S=E(\overrightarrow{\mathrm{a}}, \overrightarrow{\mathrm{b}})-E\left(\overrightarrow{\mathrm{a}}, \overrightarrow{\mathrm{b}}^{\prime}\right)+E\left(\overrightarrow{\mathrm{a}}^{\prime}, \overrightarrow{\mathrm{b}}\right)+E\left(\overrightarrow{\mathrm{a}}^{\prime}, \overrightarrow{\mathrm{b}}^{\prime}\right)
$$

involves four measurements in four various orientations. On the other hand, for suitable sets of orientations, ${ }^{4}$ the quantum mechanical predictions can reach the values $S= \pm 2 \sqrt{2}$, in clear contradiction with (2): Quantum mechanics cannot be completed by an underlying structure such as "realistic local theories."

Several experiments with increasing accuracy have been performed, and they clearly favor quantum mechanics. ${ }^{3,5}$ Unfortunately, none allowed a direct test using inequalities (2), since none followed the scheme of Fig. 1 closely enough. Some experiments were performed with pairs of pho- \pm 1 ; for photons, a measurement along $\vec{a}$ yields the result +1 if the polarization is found parallel to $\vec{a}$, and -1 if the polarization is found perpendicular. For a singlet state, quantum mechanics predicts some correlation between such measurements on the two particles. Let us denote by $P_{ \pm \pm}(\vec{a}, \vec{b})$ the probabilities of obtaining the result \pm 1 along $\vec{a}$ (particle 1 ) and \pm 1 along $\vec{b}$ (particle 2). The quantity

tons (or of protons). But no efficient analyzers are available at such energies, and the results that would have been obtained with ideal polarizers are deduced indirectly from Compton scattering experiments. The validity of such a procedure in the context of Bell's theorem has been criticized. $^{3,6}$

There are also experiments with pairs of lowenergy photons emitted in atomic radiative cascades. True polarizers are available in the visible range. However, all previous experiments involved single-channel analyzers, transmitting one polarization $(\vec{a}$ or $\vec{b})$ and blocking the orthog-

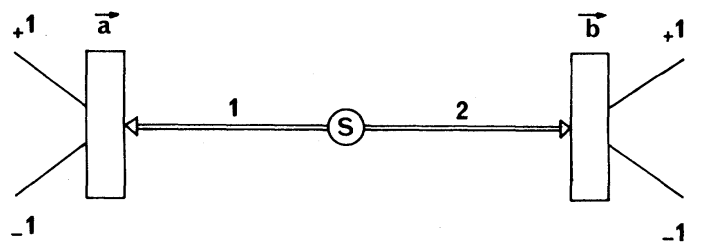

FIG. 1. Einstein-Podolsky-Rosen-Bohm gedankenexperiment. Two-spin- $\frac{1}{2}$ particles (or photons) in a singlet state (or similar) separate. The spin components (or linear polarizations) of 1 and 2 are measured along $\vec{a}$ and $\vec{b}$. Quantum mechanics predicts strong correlations between these measurements. 
onal one. The measured quantities were thus only the coincidence rates in +1 channels: $R_{++}(\overrightarrow{\mathrm{a}}$, $\vec{b})$. Several difficulties then arise ${ }^{3}$ as a result of the very low efficiency of the detection system (the photomultipliers have low quantum efficiencies and the angular acceptance is small). The measurements of polarization are inherently incomplete: When a pair has been emitted, if no count is obtained at one of the photomultipliers, there is no way to know whether it has been missed by the (low-efficiency) detector or whether it has been blocked by the polarizer (only the latter case would be a real polarization measurement). Thus, coincidence counting rates such as $R_{+-}(\overrightarrow{\mathrm{a}}, \overrightarrow{\mathrm{b}})$ or $R_{--}(\overrightarrow{\mathrm{a}}, \overrightarrow{\mathrm{b}})$ cannot be measured directly. It is nevertheless possible to derive from the experimental data numerical quantities which can (according to quantum mechanics) possibly violate Bell-type inequalities. For this purpose, one has to resort to auxiliary experiments, where coincidence rates are measured with one or both polarizers removed. Some reasoning, with a few additional-and very natural -assumptions (such as the "no-enhancement" assumption of Clauser and Horne ${ }^{7}$ ), then allows one to obtain actually operational inequalities.

In this Letter, we report the results of an experiment following much more closely the ideal

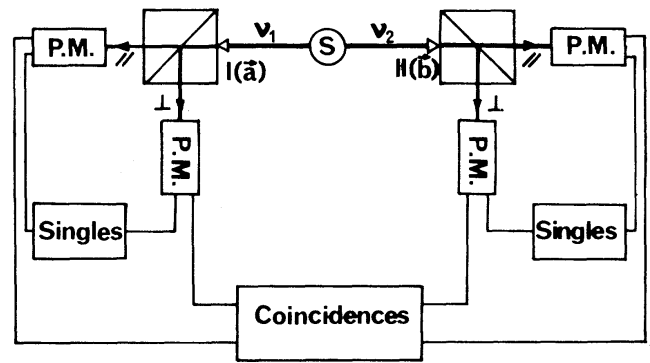

FIG. 2. Experimental setup. Two polarimeters I and II, in orientations $\vec{a}$ and $\vec{b}$, perform true dichotomic measurements of linear polarization on photons $\nu_{1}$ and $\nu_{2}$. Each polarimeter is rotatable around the axis of the incident beam. The counting electronics monitors the singles and the coincidences.

scheme of Fig. 1. True dichotomic polarization measurements on visible photons have been performed by replacing ordinary polarizers by twochannel polarizers, separating two orthogonal linear polarizations, followed by two photomultipliers (Fig. 2). The polarization measurements then become very similar to usual SternGerlach measurements for spin- $\frac{1}{2}$ particles. ${ }^{8}$

Using a fourfold coincidence technique, we measure in a single run the four coincidence rates $R_{ \pm \pm}(\overrightarrow{\mathrm{a}}, \overrightarrow{\mathrm{b}})$, yielding directly the correlation coefficient for the measurements along $\vec{a}$ and $\vec{b}$ :

$$
E(\overrightarrow{\mathrm{a}}, \overrightarrow{\mathrm{b}})=\frac{R_{++}(\overrightarrow{\mathrm{a}}, \overrightarrow{\mathrm{b}})+R_{--}(\overrightarrow{\mathrm{a}}, \overrightarrow{\mathrm{b}})-R_{+-}(\overrightarrow{\mathrm{a}}, \overrightarrow{\mathrm{b}})-R_{-+}(\overrightarrow{\mathrm{a}}, \overrightarrow{\mathrm{b}})}{R_{++}(\mathrm{a}, \mathrm{b})+R_{--}(\mathrm{a}, \mathrm{b})+R_{+-}(\mathrm{a}, \mathrm{b})+R_{-+}(\mathrm{a}, \mathrm{b})} .
$$

It is then sufficient to repeat the same measurements for three other choices of orientations, and inequalities (2) can directly be used as a test of realistic local theories versus quantum mechanics. This procedure is sound if the measured values (3) of the correlation coefficients can be taken equal to the definition (1), i.e., if we assume that the ensemble of actually detected pairs is a faithful sample of all emitted pairs. This assumption is highly reasonable with our very symmetrical scheme, where the two measurement results +1 and -1 are treated in the same way (the detection effiencies in both channels of a polarizer are equal). All data are collected in very similar experimental conditions, the only changes being rotations of the polarizers.

Such a procedure allows us not only to suppress possible systematic errors (e.g., changes occur ring when removing the polarizers) but also to control more experimental parameters. For instance, we have checked that the sum of the coincidence rates of one photomultiplier with both photomultipliers on the other side is constant. We have also observed that the sum of the four coincidence rates $R_{ \pm \pm}(\overrightarrow{\mathrm{a}}, \overrightarrow{\mathrm{b}})$ is constant when changing the orientations; thus the size of the selected sample is found constant.

We have used the high-efficiency source previously described. ${ }^{5} \mathrm{~A}(J=0) \rightarrow(J=1) \rightarrow(J=0)$ cas cade in calcium-40 is selectively excited by twophoton absorption, with use of two single-mode lasers. Pairs of photons (at wavelengths $\lambda_{1}$ $=551.3 \mathrm{~nm}$ and $\lambda_{2}=422.7 \mathrm{~nm}$ ) correlated in polarization are emitted at a typical rate of $5 \times 10^{7}$ $\mathrm{s}^{-1}$. The polarizers are polarizing cubes (Fig. 2) made of two prisms with suitable dielectric thin films on the sides stuck together; the faces are antirefelction coated. Cube I transmits light polarized in the incidence plane onto the active hypotenuse (parallel polarization, along $\vec{a}$ ) while it reflects the orthogonal polarization (perpendicular polarization). Cube II works similarly. For actual polarizers we define transmission and re- 
flection coefficients: $T^{\|}$and $R^{\perp}$ are close to 1 , while $T^{\perp}$ and $R^{\|}$are close to 0 . The measured values of our devices are $T_{1}{ }^{\prime \prime}=R_{1}{ }^{\perp}=0.950$ and $T_{1}{ }^{\perp}=R_{1}{ }^{\prime \prime}=0.007$ at $\lambda_{1} ; T_{2}{ }^{11}=R_{2}{ }^{\perp}=0.930$ and $T_{2}{ }^{\perp}$ $=R_{2}{ }^{\prime \prime}=0.007$ at $\lambda_{2}$ (all values are \pm 0.005 ). Each polarizer is mounted in a rotatable mechanism holding two photomultipliers; we call the ensemble a polarimeter. The gains of the two photomultipliers are adjusted for the equality of the counting detection efficiencies in both channels of a polarimeter $\left(2 \times 10^{-3}\right.$ at $422 \mathrm{~nm}, 10^{-3}$ at 551 $\mathrm{nm}$ ). Typical single rates (over $10^{4} \mathrm{~s}^{-1}$ ) are high compared with dark rates $\left(10^{2} \mathrm{~s}^{-1}\right)$. Wavelength filters at 422 or $551 \mathrm{~nm}$ are mounted in front of each photomultiplier. The fourfold coincidence electronics includes four overlap-type coincidence circuits. Each coincidence window, about $20 \mathrm{~ns}$ wide, has been accurately measured. Since they are large compared to the lifetime of the intermediate state of the cascade ( $5 \mathrm{~ns}$ ) all true coincidences are registered. We infer the accidental coincidence rates from the corresponding single rates, knowing the widths of the windows. This method is valid with our very stable source, and it has been checked by comparing it with the methods of Ref. 5, using delayed coincidence channels and/or a time-to-amplitude converter. By subtraction of these accidental rates (about $10 \mathrm{~s}^{-1}$ ) from the total rates, we obtain the true coincidence rates $R_{ \pm \pm}(\overrightarrow{\mathrm{a}}, \overrightarrow{\mathrm{b}})$ (actual values are in the range $0-40 \mathrm{~s}^{-1}$, depending on the orientations). A run lasts $100 \mathrm{~s}$, and $E(\vec{a}, \vec{b})$ derived from Eq. (3) is measured with a typical statistical accuracy of \pm 0.02 (the sum of the four coincidence rates is typically $80 \mathrm{~s}^{-1}$ ).

It is well known that the greatest conflict be tween quantum mechanical predictions and the inequalities (2) is expected for the set of orientations $(\vec{a}, \vec{b})=\left(\vec{b}, \vec{a}^{\prime}\right)=\left(\vec{a}^{\prime}, \vec{b}^{\prime}\right)=22.5^{\circ}$ and $\left(\vec{a}, \vec{b}^{\prime}\right)$ $=67.5^{\circ}$. Five runs have been performed at each of these orientations; the average yields

$$
S_{\text {expt }}=2.697 \pm 0.015 \text {. }
$$

The indicated uncertainty is the standard deviation accounting for the Poisson law in photon counting. The impressive violation of inequalities (2) is $83 \%$ of the maximum violation predicted by quantum mechanics with ideal polar izers (the largest violation of generalized Bell's inequalities previously reported was $55 \%$ of the predicted violation in the ideal case ${ }^{5}$ ).

With symmetrical polarimeters, quantum mech-

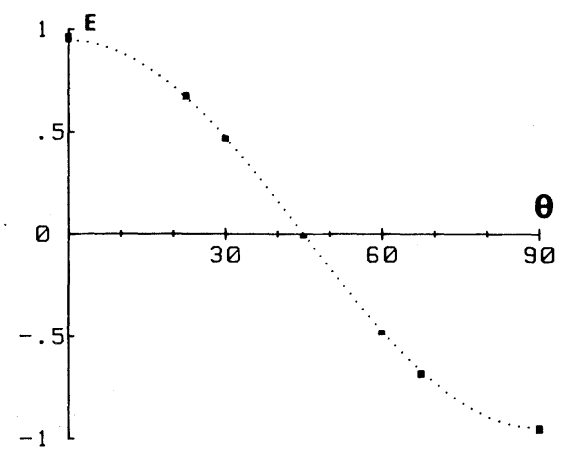

FIG. 3. Correlation of polarizations as a function of the relative angle of the polarimeters. The indicated errors are \pm 2 standard deviations. The dotted curve is not a fit to the data, but quantum mechanical predictions for the actual experiment. For ideal polarizers, the curve would reach the values \pm 1 .

anics predicts

$$
E(\overrightarrow{\mathrm{a}}, \overrightarrow{\mathrm{b}})=F \frac{\left(T_{1}{ }^{\prime \prime}-T_{1}{ }^{\perp}\right)\left(T_{2}{ }^{\prime \prime}-T_{2}{ }^{\perp}\right)}{\left(T_{1}{ }^{11}+T_{1}{ }^{\perp}\right)\left(T_{2}{ }^{11}+T_{2}{ }^{\perp}\right)} \cos 2(\overrightarrow{\mathrm{a}}, \overrightarrow{\mathrm{b}}) .
$$

$(F=0.984$ in our case; it accounts for the finite solid angles of detection.) Thus, for our experiment,

$$
S_{\mathrm{QM}}=2.70 \pm 0.05 \text {. }
$$

The indicated uncertainty accounts for a slight lack of symmetry between both channels of a polarimeter: We have found a variation of $\pm 1 \%$ of the detection efficiencies when rotating the polarimeters. This spurious effect has been explained as small displacements of the light beam impinging onto the photocathode. The effect of these variations on the quantum mechanical predictions has been computed, and cannot create a variation of $S_{\mathrm{QM}}$ greater than $2 \% .^{\circ}$

Figure 3 shows a comparison of our results with the predictions of quantum mechanics. Here, for each relative orientation $\theta=(\vec{a}, \vec{b})$, we have averaged several measurements in different $a b-$ solute orientations of the polarimeters; this procedure averages out the effect of the slight variations of the detection efficiencies with orientation. The agreement with quantum mechanics is better than $1 \%$.

In conclusion, our experiment yields the strongest violation of Bell's inequalities ever achieved, and excellent agreement with quantum mechanics. Since it is a straightforward transposition of the ideal Einstein-Podolsky-Rosen-Bohm scheme, 
the experimental procedure is very simple, and needs no auxiliary measurements as in previous experiments with single-channel polarizers. We are thus led to the rejection of realistic local theories if we accept the assumption that there is no bias in the detected samples: Experiments support this natural assumption.

Only two loopholes remain open for advocates of realistic theories without action at a distance. ${ }^{10}$ The first one, exploiting the low efficiencies of detectors, could be ruled out by a feasible experiment. ${ }^{11}$ The second one, exploiting the static character of all previous experiments, could also be ruled out by a "timing experiment" with variable analyzers ${ }^{12}$ now in progress.

The authors acknowledge many valuable discussions with F. Laloë about the principle of this experiment. They are grateful to C. Imbert who sponsors this work.

${ }^{1}$ A. Einstein, B. Podolsky, and N. Rosen, Phys. Rev. 47, 777 (1935); D. J. Bohm, Quantum Theory (PrenticeHall, Englewood Cliffs, N.J., 1951).

2J. S. Bell, Physics (N.Y.) 1, 195 (1965).

${ }^{3}$ J. F. Clauser and A. Shimony, Rep. Prog. Phys. 41,
1981 (1978). This paper is an exhaustive review of this question.

${ }^{4}$ J. F. Clauser, M. A. Horne, A. Shimony, and R. A. Holt, Phys. Rev. Lett. 23, 880 (1969).

${ }^{5}$ A. Aspect, P. Grangier, and G. Roger, Phys. Rev. Lett. 47,460 (1981).

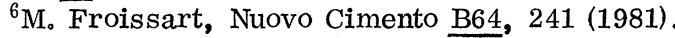

7J. F. Clauser and M. A. Horne, Phys. Rev. D $\underline{10}$, 526 (1974).

${ }^{8}$ Such an experimental scheme has been proposed and discussed by several authors: A. Shimony, in Foundations of Quantum Mechanics, edited by B. d'Espagnat (Academic, New York, 1972); F. Laloë, private communication; A. Garuccio and V. Rapisarda, Nuovo Cimento A65, 269 (1981); see also Ref. 7. A similar experiment has been undertaken by V. Rapisarda et al.

${ }^{9}$ Alternatively, this lack of symmetry can be taken into account in generalized Bell's inequalities similar to inequalities (2). (The demonstration will be published elsewhere.) In our case, the inequalities then become $|S| \leqslant 2.08$. The violation is still impressive.

${ }^{10} \mathrm{As}$ in our previous experiments, the polarizers are separated by $13 \mathrm{~m}$. The detection events are thus spacelike separated, and we eliminate the loophole considered by L. Pappalardo and V. Rapidarda, Lett. Nuovo Cimento 29, 221 (1980).

${ }^{11}$ T. K. Lo and A. Shimony, Phys. Rev。A 23,3003 (1981)。

${ }^{12}$ A. Aspect, Phys。Rev。D $\underline{14}, 1944$ (1976).

\title{
Hopping Mechanism Generating $1 / f$ Noise in Nonlinear Systems
}

\author{
F. T. Arecchi and F. Lisi \\ University of Florence and Istituto Nazionale di Ottica, Florence, Italy \\ (Received 17 February 1982)
}

\begin{abstract}
It is shown experimentally that a bistable system is driven by a suitable external modulation to a region of random intermittency which displays a low-frequency power-law divergence. This low-frequency divergence is associated with a topological alternation between two strange attractors in phase space, either unsymmetric or fully symmetric depending on whether the two potential valleys are differently or equally located. This picture seems sufficiently general to apply to most cases of low-frequency noise currently reported.
\end{abstract}

PACS numbers: $05.40 .+\mathrm{j}, 05.70 . \mathrm{Ln}$

We show evidence of a mechanism responsible for low-frequency excess noise in a nonlinear system. The mechanism seems to be sufficiently general to be a possible explanantion for many physical cases where excess, or $1 / f$, noise appears, even though we do not pretend to claim full universality.

In order to study the routes to chaos in nonlinear nonequilibrium physical systems we have built a suitable electronic oscillator with a cubic non- linearity imposed by a selected field-effect transistor device and driven by an external modulation. The internal force law corresponds to a twovalley potential. The dynamical equation for the normalized oscillator output $x(t)$ is

$$
\frac{d^{2} x}{d \tau^{2}}+k \frac{d x}{d \tau}-x+4 x^{3}=A \cos \omega \tau,
$$

where $\tau=\omega_{0} t, \omega_{0}$ is the oscillator frequency, $\omega$ is the modulation frequency normalized to $\omega_{0}$, 